\title{
Исследование структурных и люминесцентных свойств гетероструктур InAs/GaAs с легированными Bi потенциальными барьерами
}

\author{
(C) А.С. Пащенко ${ }^{1,2}$, Л.С. Лунин ${ }^{1,2}$, С.Н. Чеботарев ${ }^{1,2}$, М.Л. Лунина $^{1}$ \\ ${ }^{1}$ Южный научный центр Российской академии наук, \\ 344006 Ростов-на-Дону, Россия \\ ${ }^{2}$ Южно-Российский государственный политехнический университет (НПИ) им. М.И. Платова, \\ 346428 Новочеркасск, Россия \\ E-mail: as.pashchenko@gmail.com
}

(Получена 10 мая 2017 г. Принята к печати 31 мая 2017 г.)

Исследовано влияние Bi в барьерных слоях GaAs на структурные и оптические свойства гетероструктур InAs/GaAs с квантовыми точками. Методами атомно-силовой микроскопии и рамановской спектроскопии установлено, что введение Вi до 5 ат\% в GaAs приводит к снижению плотности квантовых точек InAs c $1.58 \cdot 10^{10}$ до $0.93 \cdot 10^{10} \mathrm{~cm}^{-2}$. Эффект обусловлен уменьшением величины рассогласования параметра кристаллических решеток в гетероинтерфейсе InAs/GaAsBi. При этом отмечается увеличение высоты квантовых точек InAs, вероятно, вызванное возрастанием поверхностной диффузии In в процессе роста на поверхности GaAsBi. Анализ люминесцентных свойств показал, что легирование висмутом потенциальных барьеров GaAs сопровождается красным смещением пика излучения квантовых точек InAs и уменьшает его ширину.

DOI: $10.21883 /$ FTP.2018.06.45919.8635

\section{1. Введение}

Создание наногетероструктур $\mathrm{A}^{\mathrm{III}} \mathrm{B}^{\mathrm{V}}$ с квантовыми точками (KT/QD) открывает возможность развития инжекционных полупроводниковых лазеров [1-5], солнечных элементов [6-9] и фотоприемных устройств $[10,11]$ нового поколения. Локализация фотогенерированных носителей заряда в квантовой точке по трем направлениям способствует уменьшению их термогенерации и приводит к снижению темнового тока $[11,12]$. Примером таких гетероструктур могут быть InAs/GaAs [13-15], Ge/Si [16] и другие [17-24]. Рост самоорганизованных квантовых точек происходит в режиме Странского-Крастанова, когда смачивающий подложку слой является упругонапряженным. Для создания условий к переходу от 2D к 3D росту гетеропара InAs/GaAs является идеальной, но имеет ограничения для ряда функциональных применений, например, варьирования спектра поглощения фотодетекторов инфракрасного излучения. Известно, что висмут является изовалентной примесью и образует тройные, четверные и пятерные твердые растворы с соединениями $\mathrm{A}^{\mathrm{III}} \mathrm{B}^{\mathrm{V}}$ [25-29]. В 2002 г. были успешно легированы висмутом слои твердых растворов GaAsP в поле температурного градиента [30]. В 2003 г. впервые успешно выращены слои GaAsBi c фракцией $\mathrm{Bi} 3 \%$ методом молекулярно-лучевой эпитаксии [31]. Введение $\mathrm{Bi}$ в кристаллическую решетку $\mathrm{GaAs}$ позволяет изменять величину рассогласования параметра решетки при росте квантовых точек InAs и, кроме того, приводит к появлению локализованных состояний в запрещенной зоне GaAs вблизи потолка валентной зоны. Возникающие напряжения в матрице GaAsBi должны оказывать влияние на поверхностную плотность, геометрические размеры квантовых точек InAs и, следовательно, на люминесцентные свойства гетероструктур InAs/GaAs. Также известны применения для этих целей и других материалов: GaAsSb [32], InGaAs [33], InGaAsN [34,35], $\mathrm{GaAsNSb}[36,37]$. Необходимость изучения легирования $\mathrm{Bi}$ потенциальных барьеров GaAs обусловлена еще и тем, что из-за расщепления валентной зоны слои GaAsBi обладают собственной проводимостью $p$-типа. Это имеет перспективу при создании $p-i-n$-структур с квантовыми точками. В этой связи изучение влияния висмута на структурные и оптические свойства гетероструктур InAs/GaAs является актуальной задачей.

Целью данной работы является исследование влияния Bi в барьерных слоях GaAs на структурные и люминесцентные свойства гетероструктур InAs/GaAs c квантовыми точками.

\section{2. Методика эксперимента}

Для исследований были выращены экспериментальные образцы с потенциальными барьерами $\mathrm{GaAs}_{1-x} \mathrm{Bi}_{x}$ с фракцией $\mathrm{Bi} x=1,3$ и 5 ат\%. Один экспериментальный образец содержал нелегированные барьеры GaAs для проведения сравнения. Выращивание экспериментальных образцов осуществлялось с использованием одного из методов физического распыления - ионнолучевого [38-43]. В качестве источника материала для выращивания слоев $\mathrm{GaAs}_{1-x} \mathrm{Bi}_{x}$ были использованы предварительно синтезированные поликристаллические мишени. Их изготовление осуществлялось следующим образом. Рассчитывался состав шихты из взвешенной навески компонентов в соответствии с составом твердой 
фазы готовой мишени. Общая масса расплава рассчитывалась в соответствии с объемом предполагаемого слитка поликристаллической мишени. После химической обработки компоненты шихты помещались в контейнер из упрочненного графита. В резистивной печи контейнер с шихтой нагревался и выдерживался несколько минут выше температуры плавления самого тугоплавкого соединения GaAs $\left(1250^{\circ} \mathrm{C}\right)$. Затем следовало резкое отключение нагревательных элементов и происходила кристаллизация исходного расплава. Полученный поликристаллический слиток разрезался на пластины толщиной 2 мм.

Выращивание гетероструктур с квантовыми точками проводилось в экспериментальной установке ионнолучевого осаждения. Эксперименты по осаждению выполнялись на подложки арсенида галлия (GaAs) с кристаллографической ориентацией (100). Осаждение материала осуществлялось прямым распылением мишени пучком ионов $\mathrm{Ar}^{+}$. Калибровочные зависимости коэффициента распыления InAs и GaAs от энергии пучка, угла наклона и плотности тока приведены в работах [42-44]. Первым выращивался буферный слой $p^{+}$-GaAs толщиной 500 нм при температуре $535^{\circ} \mathrm{C}$. Энергия ионов в пучке составляла $450 \mathrm{~B}$ при плотности ионного тока $5 \cdot 10^{-4} \mathrm{~A} / \mathrm{cm}^{2}$. Затем выдерживалась пауза 15 с и снижалась температура до $450^{\circ} \mathrm{C}$. Рост слоев квантовых точек InAs проводился при ускоряющем напряжении ионов 250 В и плотности ионного тока $1.2 \cdot 10^{-5} \mathrm{~A} / \mathrm{cm}^{2}$. Для всех экспериментальных образцов скорость роста квантовых точек InAs составляла $0.1 \mathrm{MC} / \mathrm{c}$ (MC монослой). Выращивание барьерных слоев $\mathrm{GaAs}_{1-x} \mathrm{Bi}_{x}$ проводилось при температуре $360^{\circ} \mathrm{C}$ для устранения эффекта десорбции Ві с поверхности роста [45-47]. Далее формировался пассивирующий слой $n^{+}$-GaAs (200 нм).

Исследования морфологии поверхности выращенных гетероструктур и их кристаллического качества проводились на атомно-силовом микроскопе Solver HV и рамановском спектрометре Renishaw InVia Raman соответственно.

Изучение люминесцентных свойств выращенных гетероструктур выполнялось методом фотолюминесценции (ФЛ) в спектральном диапазоне 800-1500 нм при температуре $T=90 \mathrm{~K}$. Источником оптического излучения служил инжекционный лазер с длиной волны 402 нм. Регистрацию фотолюминесцентного сигнала осуществляли с помощью монохроматора МДР-23 и фотоприемного устройства с германиевым фотодиодом ФДГ-3600. Защита входной щели монохроматора от попадания лазерного излучения осуществлялась с помощью оптического фильтра ЖС-12.

\section{3. Результаты и их обсуждение}

\section{1. Исследования поверхности методом атомно-силовой микроскопии}

Исследована морфология поверхности с квантовыми точками InAs, выращенными с использованием различных потенциальных барьеров: GaAs и $\mathrm{GaAs}_{0.95} \mathrm{Bi}_{0.05}$.

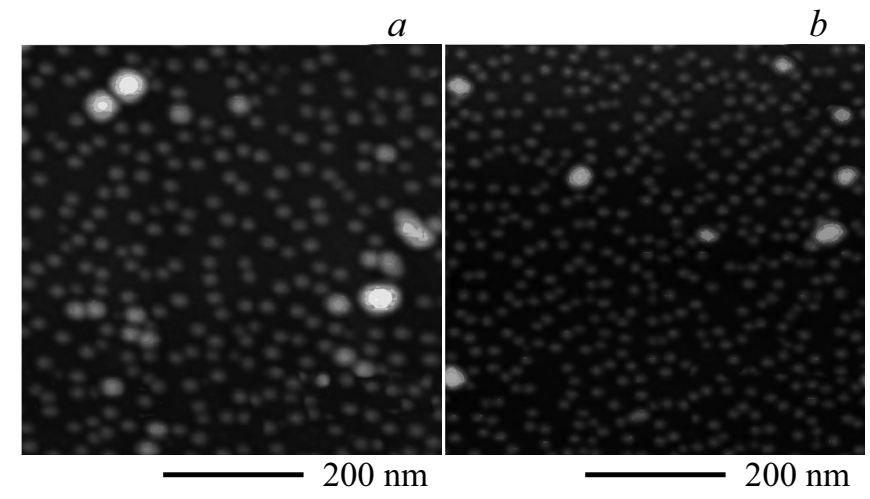

Рис. 1. АСМ-изображения поверхности: $a-\mathrm{KT}$ InAs на $\mathrm{GaAs}_{0.95} \mathrm{Bi}_{0.05} ; b-\mathrm{KT}$ InAs на GaAs.

На рис. 1 наблюдается снижение плотности КТ InAs при их росте на поверхности $\mathrm{GaAs}_{0.95} \mathrm{Bi}_{0.05}$. Выполненный статистический анализ инструментом Threshold в программе Image Analysis 2.1.2 показал, что плотность КТ в гетеросистеме InAs/ $\mathrm{GaAs}_{0.95} \mathrm{Bi}_{0.05}$ составила $0.93 \cdot 10^{10} \mathrm{~cm}^{-2}$, для InAs/GaAs $-1.58 \cdot 10^{10} \mathrm{~cm}^{-2}$. В то же время добавление $\mathrm{Bi}$ в матрицу $\mathrm{GaAs}$ сопровождается увеличением среднего геометрического размера КТ InAs c 14-16 до 20 нм. Результаты АCМ-исследований могут быть объяснены повышением скорости поверхностной диффузии In в процессе осаждения квантовых точек на поверхность $\mathrm{GaAs}_{0.95} \mathrm{Bi}_{0.05}$. Об этом также свидетельствует увеличение высоты КТ в гетеросистеме $\mathrm{InAs} / \mathrm{GaAs}_{0.95} \mathrm{Bi}_{0.05}$. Следовательно, наличие $\mathrm{Bi}$ на ростовой поверхности GaAs изменяет кинетику поверхностной диффузии In в процессе ионно-лучевого осаждения KT InAs. Из-за большого размера атома висмут создает в кристаллической решетке GaAs упругие деформации и таким образом влияет на самоорганизацию квантовых точек.

\section{2. Рамановская спектроскопия}

Поскольку висмут является изовалентной примесью для соединений $\mathrm{A}^{\mathrm{III}} \mathrm{B}^{\mathrm{V}}$, то он замещает атомы элемента V группы. В данном случае Ві замещает атомы мышьяка. Таким образом, кристаллическая решетка $\mathrm{GaAs}_{1-x} \mathrm{Bi}_{x}$ представляет собой решетку типа цинковой обманки. В состоянии равновесия в соответствии с правилом отбора для нее разрешены следующие моды: продольных оптических (LO) фононов и продольных акустических (LA) фононов. На рис. 2 приведены результаты исследования гетерограниц InAs/GaAs и $\operatorname{InAs} / \mathrm{GaAs}_{1-x} \mathrm{Bi}_{x}$. Видно, что кроме разрешенных мод наблюдаются и запрещенные поперечные оптические (TO) моды для объемных GaAs- и GaBi-подобных оптических фононов при 268 и $182 \mathrm{~cm}^{-1}$ соответственно. Их появление может быть объяснено изменением правила отбора при рамановском рассеянии в кристаллической решетке типа цинковой обманки, вызванного деформациями кристаллической решетки при внедре- 


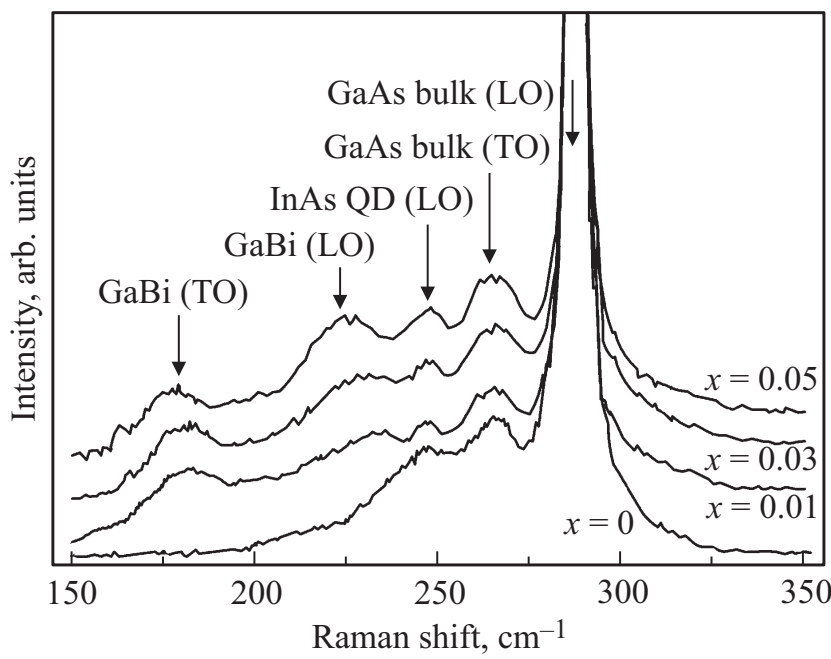

Рис. 2. Спектры рамановского рассеяния для гетероструктур InAs/GaAs и InAs/ $\mathrm{GaAs}_{1-x} \mathrm{Bi}_{x}$.

нии Bi в матрицу GaAs. B спектрах отчетливо видны продольные моды оптических фононов объемного GaAs (LO) - $283 \mathrm{~cm}^{-1}$, квантовых точек InAs (LO) $248 \mathrm{~cm}^{-1}$, GaBi на $235 \mathrm{~cm}^{-1}$. Данные рис. 2 показывают, что в диапазоне $150-250 \mathrm{~cm}^{-1}$ в спектрах образцов $\mathrm{InAs} / \mathrm{GaAs}_{1-x} \mathrm{Bi}_{x}$ проявляются эффекты, не обнаруживаемые в спектре рамановского смещения гетероструктуры InAs/GaAs. Увеличение доли висмута $x$ сопровождается рамановским смещением GaAs- и GaBi-подобных пиков в область меньших значений волнового числа. Наряду с этим проявляется слабое увеличение интенсивности пиков. Рамановское смещение пиков в спектрах гетероструктур InAs/GaAs ${ }_{1-x} \mathrm{Bi}_{x}$ относительно спектра гетероструктуры InAs/GaAs обусловлено большей степенью релаксации гетерограницы $\mathrm{InAs} / \mathrm{GaAs}_{1-x} \mathrm{Bi}_{x}$ по сравнению с InAs/GaAs за счет уменьшения величины рассогласования параметров кристаллической решетки. Полученные результаты рамановских исследований хорошо согласуются с результатами АСМ-исследований.

\section{3. Фотолюминесценция}

Исследование рекомбинационных процессов в экспериментальных образцах InAs/GaAs и InAs/GaAs ${ }_{1-x} \mathrm{Bi}_{x}$ было выполнено измерением фотолюминесценции. Для предварительной оценки снижения энергии оптического перехода при введении висмута в матрицу GaAs была рассчитана ширина запрещенной зоны $E_{g}$ объемного $\mathrm{GaAs}_{1-x} \mathrm{Bi}_{x}$ согласно выражению $[48,49]$

$$
\begin{aligned}
E_{q}\left(\mathrm{GaAs}_{1-x} \mathrm{Bi}_{x}\right)= & -0.36 x+1.42(1-x) \\
& -\frac{9.5}{1+10.4 x}(1-x) .
\end{aligned}
$$

Рассчитанные значения ширины запрещенной зоны $\mathrm{GaAs}_{1-x} \mathrm{Bi}_{x}$ для исследуемых составов приведены в таблице. Данные таблицы показывают, что внедрение
Рассчитанные значения ширины запрещенной зоны соединения $\mathrm{GaAs}_{1-x} \mathrm{Bi}_{x}$ в зависимости от состава

\begin{tabular}{c|c}
\hline$x$ & $E_{g},{ }{ }_{\mathrm{B}}$ \\
\hline 0.01 & 1.32 \\
0.03 & 1.17 \\
0.05 & 0.98
\end{tabular}

висмута в потенциальные барьеры GaAs создает структуру, в которой KT InAs находятся в квантовой яме $\mathrm{GaAs}_{1-x} \mathrm{Bi}_{x}$, ограниченной барьерами GaAs (рис. 3). Это изменение энергетической зонной диаграммы способно смещать спектр излучения ФЛ квантовых точек InAs в область длинных волн.

На рис. 4 приведены результаты исследования фотолюминесценции экспериментальных образцов при $T=90 \mathrm{~K}$. Сплошной линией показан спектр образца, в котором KT InAs ограничены барьером GaAs. Видно, что интегральный спектр состоит из трех пиков: объемного
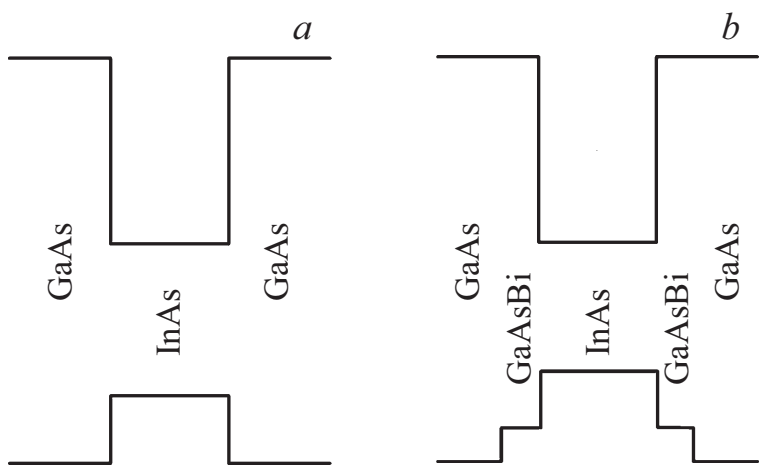

Рис. 3. Зонные диаграммы гетероструктур с барьером GaAs $(a)$ и $\mathrm{GaAsBi}(b)$.

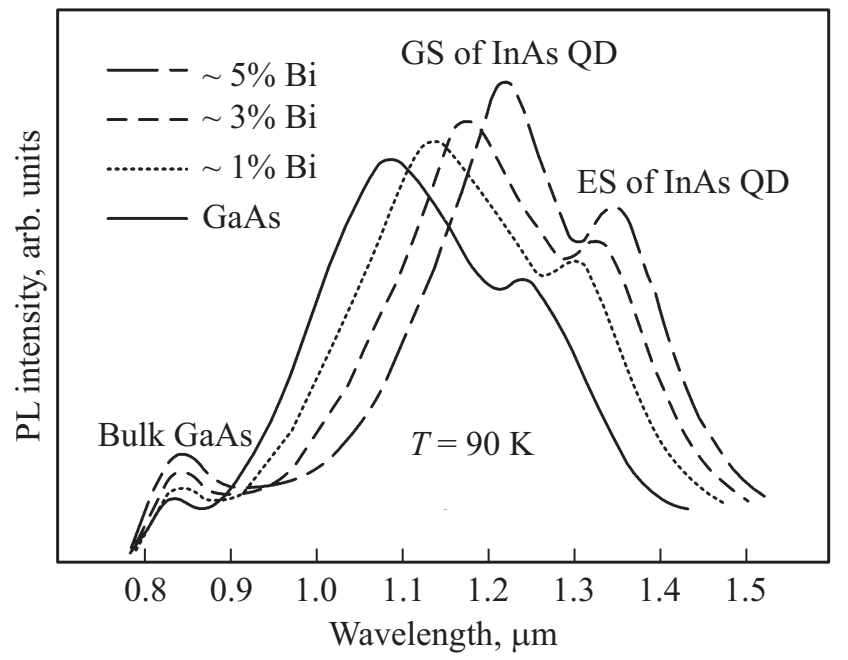

Рис. 4. Спектры фотолюминесценции (PL) гетероструктур InAs/GaAsBi c различным содержанием Bi в потенциальных барьерах. 
арсенида галлия (830 нм), пика GS (1082 нм) основных состояний в КТ и пика ES (1236 нм) возбужденных состояний в КТ.

Введение в матрицу GaAs висмута в количестве $x=1-5 \%$ сопровождается красным смещением пиков ФЛ квантовых точек InAs, что обусловлено релаксацией упругих напряжений на гетерогранице InAs/ $\mathrm{GaAs}_{1-x} \mathrm{Bi}_{x}$. Анализ показывает уменьшение ширины спектра на половине максимума излучения (рис. 4) и возрастание интенсивности всех пиков ФЛ. Повышение доли Ві в матрице GaAs до $x=0.05$ сопровождается смещением пика GS квантовых точек InAs в область длинных волн на 100 мэВ. При этом уменьшается полуширина пика GS. Наблюдаемый эффект свидетельствует о повышении кристаллического качества гетерограницы InAs/GaAs ${ }_{1-x} \mathrm{Bi}_{x}$ и хорошо согласуется с результатами $\mathrm{ACM}$ и рамановской спектроскопии.

Рассмотрим особенности рекомбинационных процессов в гетероструктурах InAs/ $\mathrm{GaAs}_{1-x} \mathrm{Bi}_{x}$. Несмотря на снижение плотности квантовых точек, отмечается увеличение интенсивности излучательной рекомбинации $\mathrm{c}$ повышением содержания висмута в арсениде галлия. Данный эффект объясняется тем, что висмут приводит к расщеплению валентной зоны GaAs, смещая энергетический максимум тяжелых дырок вглубь запрещенной зоны, из-за чего она уменьшается пропорционально концентрации висмута. Это приводит к изменению процесса генерации носителей заряда при возбуждении ФЛ и изменению механизма их излучательной рекомбинации. Снижение плотности квантовых точек InAs в случае с матрицей $\mathrm{GaAs}_{1-x} \mathrm{Bi}_{x}$ не является отрицательным фактором. Находясь на более удаленном расстоянии друг от друга, квантовые точки способны образовывать более узкую энергетическую подзону в зоне проводимости, что дает предпосылки для использования этого эффекта при создании многоцветных фотодетекторов (multicolor photodetectors), чувствительных к разным группам длин волн [50]. Механизмами этого могут стать внешнее смещение электрическим полем и степень оптического возбуждения гетероструктуры InAs/ $\mathrm{GaAs}_{1-x} \mathrm{Bi}_{x}$.

\section{4. Заключение}

Исследованы структурные свойства гетероинтерфейсов InAs/GaAs и InAs/ $\mathrm{GaAs}_{1-x} \mathrm{Bi}_{x}$. Методами рамановского смещения и АСМ установлено, что ионнолучевое осаждение квантовых точек InAs на поверхность $\mathrm{GaAs}_{1-x} \mathrm{Bi}_{x}$ сопровождается увеличением поверхностной диффузии In. Наблюдаемое рамановское смещение пиков в спектрах гетероструктур $\mathrm{InAs} / \mathrm{GaAs}_{1-x} \mathrm{Bi}_{x}$ относительно спектра нелегированной гетероструктуры $\mathrm{InAs} / \mathrm{GaAs}$ обусловлено большей степенью релаксации гетерограницы InAs/ $\mathrm{GaAs}_{1-x} \mathrm{Bi}_{x}$ за счет меньшего рассогласования параметров кристаллических решеток.

Методом фотолюминесценции установлено, что повышение доли $\mathrm{Bi}$ в матрице $\mathrm{GaAs}$ до $x=0.05$ приводит к красному смещению пика основных состояний электронов квантовых точек InAs. При этом уменьшается полуширина пика. Подобное поведение косвенно свидетельствует о повышении кристаллического качества гетероинтерфейса InAs/ $\mathrm{GaAs}_{1-x} \mathrm{Bi}_{x}$. Однако полностью механизмы рекомбинации носителей заряда в выращенных гетероструктурах не раскрыты. Это станет целью наших дальнейших исследований.

Отметим, что обнаруженный эффект снижения плотности квантовых точек не является отрицательным. Полученные результаты могут быть использованы в разработке многоцветных фотодетекторов. В таком случае изменение спектра чувствительности производится не формой и плотностью квантовых точек, а степенью легирования висмутом потенциальных барьеров GaAs.

Работа выполнена в рамках реализации государственного задания ЮНЦ РАН (проект № 01201354240), а также при финансовой поддержке РФФИ (в рамках научных проектов № 16-38-60127 мол_а_дк, 16-08-01052, $16-38-00575,17-08-01206)$.

\section{Список литературы}

[1] N.V. Kryzhanovskaya, E.I. Moiseev, Y.S. Polubavkina, F.I. Zubov, M.V. Maximov, A.A. Lipovskii, M.M. Kulagina, S.I. Troshkov, V.-M. Korpijärvi, T. Niemi, R. Isoaho, M. Guina, M.V. Lebedev, T.V. Lvova, A.E. Zhukov. J. Appl. Phys., 120 (23), 233103 (2016).

[2] F.I. Zubov, N.V. Kryzhanovskaya, E.I. Moiseev, Y.S. Polubavkina, O.I. Simchuk, M.M. Kulagina, Y.M. Zadiranov, S.I. Troshkov, A.A. Lipovskii, M.V. Maximov, A.E. Zhukov. Semiconductors, 50 (10), 1408 (2016).

[3] A.E. Zhukov, A.R. Kovsh. Quant. Electron., 38 (5), 409 (2008).

[4] A.M. Nadtochiy, S.A. Mintairov, N.A. Kalyuzhnyy, S.S. Rouvimov, Y.M. Shernyakov, A.S. Payusov, M.V. Maximov, A.E. Zhukov. Semiconductors, 49 (8), 1090 (2015).

[5] G.S. Sokolovskii, E.A. Viktorov, M. Abusaa, J. Danckaert, V.V. Dudelev, E.D. Kolykhalova, K.K. Soboleva, A.G. Deryagin, I.I. Novikov, M.V. Maximov, A.E. Zhukov, V.M. Ustinov, V.I. Kuchinskii, W. Sibbett, E.U. Rafailov, T. Erneux. Appl.Phys. Lett., 106 (26), 261103 (2015).

[6] N.A. Kalyuzhnyy, S.A. Mintairov, R.A. Salii, A.M. Nadtochiy, A.S. Payusov, P.N. Brunkov, V.N. Nevedomsky, M.Z. Shvarts, A. Martí, V.M. Andreev, A. Luque. Prog. Photovolt.: Res. Appl., 24 (9), 1261 (2016).

[7] E. López, A. Datas, I. Ramiro, P.G. Linares, E. Antolín, I. Artacho, A. Martí, A. Luque, Y. Shoji, T. Sogabe, A. Ogura, Y. Okada. Solar Energy Mater. Solar Cells, 149, 15 (2016).

[8] L.S. Lunin, I.A. Sysoev, D.L. Alfimova, S.N. Chebotarev, A.S. Pashchenko. J. Surf. Inv., 5 (3), 559 (2011).

[9] S.N. Chebotarev, A.S. Pashchenko, A. Williamson, L.S. Lunin, V.A. Irkha, V.A. Gamidov. Tech. Phys. Lett., 41 (7), 661 (2015).

[10] V. Ryzhii, V. Pipa, I. Khmyrova, V. Mitin, M. Willander. Jpn. J. Appl. Phys., 39 (12 B), L1283 (2000).

[11] V. Ryzhii. J. Appl. Phys., 89 (9), 5117 (2001).

[12] J. Phillips. J. Appl. Phys., 91 (7), 4590 (2002).

[13] K. Hirakawa, S.-W. Lee, P. Lelong, S. Fujimoto, K. Hirotani, H. Sakaki. Microelectron. Eng., 63 (1), 185 (2002). 
[14] P.N. Brunkov, A.R. Kovsh, V.M. Ustinov, Yu.G. Musikhin, N.N. Ledentsov, S.G. Konnikov, A. Polimeni, A. Patané, P.C. Main, L. Eaves, C.M.A.Kapteyn. J. Electron. Mater., 28 (5), 486 (1999).

[15] S. Chakrabarti, A.D. Stiff-Roberts, X.H. Su, P. Bhattacharya, G. Ariyawansa, A.G.U. Perera. J. Phys. D: Appl. Phys., 38 (13), 2135 (2005).

[16] A.I. Yakimov, A.V. Dvurechenskii, Y.Y. Proskuryakov, A.I. Nikiforov, O.P. Pchelyakov, S.A. Teys, A.K. Gutakovskii. Appl. Phys. Lett., 75 (10), 1413 (1999).

[17] M. Kudo, T. Mishima, S. Iwamoto, T. Nakaoka, Y. Arakawa. Physica E: Low-Dimens. Syst. Nanostruct., 21 (2), 275 (2004).

[18] Z. Shuhui, W. Lu, S. Zhenwu, C. Yanxiang, T. Haitao, G. Huaiju, J. Haiqiang, W. Wenxin, C. Hong, Z. Liancheng. Nanoscale Res. Lett., 7 (1), 87 (2012).

[19] J. Böhrer, A. Krost, D. Bimberg. Appl. Phys. Lett., 64 (15), 1992 (1994).

[20] M. Hayne, R. Provoost, M.K. Zundel, Y.M. Manz, K. Eberl, V.V. Moshchalkov. Phys. Rev. B, 62 (15), 10324 (2000).

[21] M. Sugisaki, H.-W. Ren, S. Nair, K. Nishi, Y. Masumoto. Phys. Rev. B, 66 (23), 235309 (2002).

[22] E. Ribeiro, R.L. Maltez, W. Carvalho, D. Ugarte, G. MedeirosRibeiro. Appl. Phys. Lett., 81 (16), 2953 (2002).

[23] G. Liu, S.-L. Chuang, S.-H. Park. J. Appl. Phys., 88 (10), 5554 (2000).

[24] B. Liang, A. Lin, N. Pavarelli, C. Reyner, J. Tatebayashi, K. Nunna, J. He, T.J. Ochalski, G. Huyet, D.L. Huffaker. Nanotech., 20 (45), 455604 (2009).

[25] L.S. Lunin, I.A. Sysoev, L.V. Blagina, A.V. Blagin, A.A. Barannik. Inorg. Mater., 45 (8), 841 (2009).

[26] D.L. Alfimova, L.S. Lunin, M.L. Lunina. Inorg. Mater., 50 (2), 113 (2014).

[27] D.F. Reyes, F. Bastiman, C.J. Hunter, D.L. Sales, A.M. Sanchez, J.P.R. David, D. González. Nanoscale Res. Lett., 9 (1), 23 (2014).

[28] A.J. Ptak, R. France, D.A. Beaton, K. Alberi, J. Simon, A. Mascarenhas, C.-S. Jiang. J. Cryst. Growth, 338 (1), 107 (2012).

[29] A. Janotti, S.-H. Wei, S.B. Zhang. Phys. Rev. B, 65 (11), 115203 (2002)

[30] L.S. Lunin, A.V. Blagin, V.A. Ovchinnikov. J. Surf. Invest.: $X$-Ray, Synchrotron Neutron Tech., N 5, 11 (2002).

[31] S. Tixier, M. Adamcyk, T. Tiedje, S. Francoeur, A. Mascarenhas, P. Wei, F. Schiettekatte. Appl. Phys. Lett., 82 (14), 2245 (2003).

[32] H.Y. Liu, M.J. Steer, T.J. Badcock, D.J. Mowbray, M.S. Skolnick, F. Suarez, J.S. Ng, M. Hopkinson, J.P.R. David. J. Appl. Phys., 99 (4), 046104 (2006).

[33] D.P. Popescu, P.G. Eliseev, A. Stintz, K.J. Malloy. Semicond. Sci. Technol., 19 (1), 33 (2004).

[34] V.V. Mamutin, A.Y. Egorov, N.V. Kryzhanovskaya, V.S. Mikhrin, A.M. Nadtochy, E.V. Pirogov. Semiconductors, 42 (7), 805 (2008).

[35] J. Wu, W. Shan, W. Walukiewicz. Semicond. Sci. Technol., 17 (8), 860 (2002).

[36] J.G. Keizer, J.M. Ulloa, A.D. Utrilla, P.M. Koenraad. Appl. Phys. Lett., 104 (5), 053116 (2014).

[37] A.D. Utrilla, D.F. Reyes, J.M. Ulloa, D. González, T. Ben, A. Guzman, A. Hierro. Appl. Phys. Lett., 105 (4), 043105 (2014).

[38] L.S. Lunin, S.N. Chebotarev, A.S. Pashchenko, L.N. Bolobanova. Inorg. Mater., 48 (5), 439 (2012).
[39] A.S. Pashchenko, S.N. Chebotarev, L.S. Lunin, V.A. Irkha. Semiconductors, 50 (4), 545 (2016).

[40] L.S. Lunin, I.A. Sysoev, D.L. Alfimova, S.N. Chebotarev, A.S. Pashchenko. Inorg. Mater., 47 (8), 816 (2011).

[41] A.S. Pashchenko, S.N. Chebotarev, L.S. Lunin. Inorg. Mater., 51 (3), 197 (2015).

[42] S.N. Chebotarev, A.S. Pashchenko, A. Williamson, L.S. Lunin, V.A. Irkha, V.A. Gamidov. Techn. Phys. Lett., 41 (7), 661 (2015).

[43] S.N. Chebotarev, A.S. Pashchenko, V.A. Irkha, M.L. Lunina. J. Nanotech., 2016, 5340218 (2016).

[44] S.N. Chebotarev, A.S. Pashchenko, L.S. Lunin, E.N. Zhivotova, G.A. Erimeev, M.L. Lunina. Beilstein J. Nanotechnol., 8, 12 (2017).

[45] J.A. Steele, R.A. Lewis, J. Horvat, M.J.B. Nancarrow, M. Henini, D. Fan, Y.I. Mazur, M. Schmidbauer, M.E. Ware, S.-Q. Yu, G.J. Salamo. Sci. Rep., 6, 28860 (2016).

[46] R.B. Lewis, M. Masnadi-Shirazi, T. Tiedje. Appl. Phys. Lett., 101 (8), 082112 (2012).

[47] X. Lu, D.A. Beaton, R.B. Lewis, T. Tiedje, M.B. Whitwick. Appl. Phys. Lett., 92 (19), 192110 (2008).

[48] X. Lu, D.A. Beaton, R.B. Lewis, T. Tiedje, Y. Zhang. Appl. Phys. Lett., 95 (4), 041903 (2009).

[49] K. Alberi, O.D. Dubon, W. Walukiewicz, K.M. Yu, K. Bertulis, A. Krotkus. Appl. Phys. Lett., 91 (5), 051909 (2007).

[50] A. Rogalski. Opto-Electron. Rev., 16 (4), 458 (2008).

Редактор Л.В. Шаронова

\section{Study of structural and luminescent properties of InAs/GaAs heterostructures with bismuth doped potential barriers}

\author{
A.S. Pashchenko ${ }^{1,2}$, L.S. Lunin ${ }^{1,2}$, S.N. Chebotarev ${ }^{1,2}$, \\ M.L. Lunina ${ }^{1}$ \\ ${ }^{1}$ Southern Scientific Center, \\ Russian Academy of Sciences, \\ 344006 Rostov-on-Don, Russia \\ ${ }^{2}$ Platov South-Russian State Polytechnic University \\ (NPI), \\ 346428 Novocherkassk, Russia
}

Abstract The influence of $\mathrm{Bi}$ in GaAs barrier layers on the structural and optical properties of heterostructures with $\mathrm{InAs} / \mathrm{GaAs}$ dots was investigated. By using the atomic force microscopy and Raman spectroscopy it was determined that introduction of Bi up to $5 \mathrm{at} \%$ in GaAs leads to decrease in density of InAs quantum dots from $1.58 \cdot 10^{10}$ to $0.93 \cdot 10^{10} \mathrm{~cm}^{-2}$. The effect was due to a decrease in the value of the crystal lattice parameters mismatch at the InAs/GaAsBi heterointerface. Thus, an increase in the height of the InAs quantum dots was observed probably due to an increase in In diffusion during the growth process on the GaAsBi surface. Analysis of the luminescent properties showed that doping potential barriers with bismuth was accompanied by a red shift of the peak emission of InAs quantum dots and reduced its width. 